\title{
Cooperative Spectrum Sensing In Cognitive Radio Networks Using Distinct Coding Techniques
}

\author{
R.saranya ${ }^{1}$, Mrs.K.Shanthalakshmi ${ }^{2}$ \\ 1. M.E communication systems student, Department of Electronics And Communication \\ 2. Assoc.professor, Department of Electronics And Communication Adhiyamaan College of Engineering, \\ Hosur-635109.
}

\begin{abstract}
This paper considers a multiuser spectrum sharing(SS) system operating in a Rayleigh fading environment and in which every node is equipped with multiple antennas. The system employs orthogonal space-time block coding at the secondary users. Under such a framework, the average capacity and error performance under a peak interference constraint are first analyzed. For a comparison purpose, an analysis of the transmit antenna selection scheme is also presented. Finally, some selected numerical results are presented to corroborate the proposed analysis.
\end{abstract}

Keywords: Antenna selection, cognitive radio, multiuser diversity, space-time block coding (STBC).

\section{Introduction}

In the last several years, the spectrum sharing (SS) systems have been widely studied due to their advantage in solving the spectrum demand[1-6].For instance,reference[3] the channel capacity under a received power constraint and for different channel fading conditions. In addition reference [4], studied spectrum sharing systems with multiuser diversity in which the secondary user with the best channel condition is selected first for signal transmission. In practice, the cooperation between the secondary and primary users is loose, which results in imperfect channel state information (CSI) of the interference channel. Therefore [5][6], investigated the effect of imperfect CSI on the system capacity. So far most of the research results for SS systems focused on the single antenna case. Recently, applying the multiple-input multiple-output (MIMO) technology to cognitive radio networks has received growing interest.

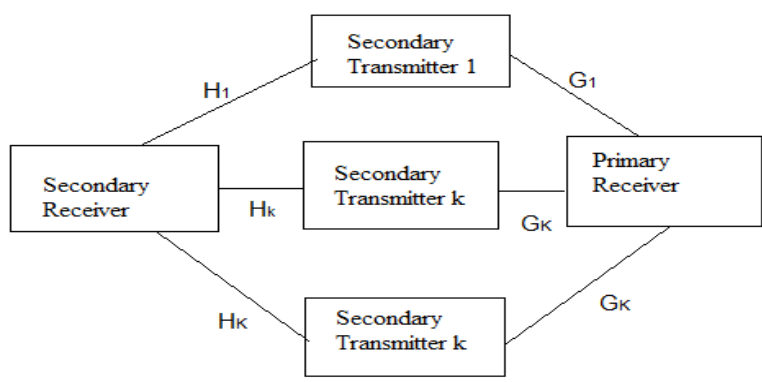

Fig.1channel model of multiuser ss OSTBC system

For instance [7] employed multiple antennas at the secondary transmitter to manage the tradeoff between throughput and interference constraint. More recently [8],the capacity limits of a multiuser multi-antenna cognitive radio (CR) network where only the base stations are equipped with multi antennas. This paper extends the results of [4] to a more general MIMO system. In fig.1 where every node is equipped with multiple antennas. We focus on orthogonal space-time block coding (OSTBC) and transmit antenna selection (TAS) CR systems with multi-user diversity and analyze the resulting average capacity and bit-error rate (BER) performance. It is known that TAS yields a better performance than OSTBC in a conventional down-link multiuser diversity MIMO system. Therefore, in this paper we want to check whether such a result is applicable to the cognitive radio environment. To investigate the diversity order of such kind of systems, the asymptotic BER expressions will be also derived.

\section{System Channel Model}

We consider an uplink multiuser SS network depicted in Fig. 1, where all the nodes are equipped with multiple antennas and a spatial diversity structure is applied at the secondary transmitter. To simplify the notations, we assume that all the nodes have the same number of antennas $N$ and ignore the interference from 
the primary transmitter to the secondary receivers. Let $\mathbf{H}_{k}$ and $\mathbf{G}_{k}$ denote the channel gain matrices from the $k^{\text {th }}$ secondary transmitter to the secondary receiver and the primary receiver, respectively.We assume that the secondary

receiver can obtain the full CSI of the channel matrix $\mathbf{H}_{k}$. All the entries of $\mathbf{H}_{k}$ and $\mathbf{G}_{k}$ are assumed independent and identically distributed (i.i.d) complex Gaussian random variables with zero mean and unit variance. To ensure that the interference constraint is satisfied at the primary receiver, we adopt the interference temperature $Q$. Notice that such a constraint requires the secondary user's access to the CSI $\mathbf{G}_{k}$ to implement the transmit power control protocol. To obtain the CSI of the interference link, some detailed channel estimation methods have been given in [1-3]. According to [4] and [5], the maximum transmitted power $P_{T}^{K}$ at the secondary transmitter can be selected according to the rule:

$P_{T}^{K}= \begin{cases}\frac{Q}{\left\|G_{K}\right\|^{2}} & \left\|G_{K}\right\|_{F}^{2}>\frac{Q}{P_{K}} \\ P_{K} & \left\|G_{K}\right\|_{F}^{2} \leq \frac{Q}{P_{K}}\end{cases}$

where $\|.\|_{\mathrm{F}}$ stands for the Frobenius norm and $\mathrm{P}_{\mathrm{k}}$ denotes the peak power of the kth secondary transmitter. As mentioned in [4], for the $Q>>\mathrm{P}_{\mathrm{k}}$ case, the effect of the interference temperature $Q$ disappears. This makes the performance analysis for the secondary system dependent only on the peak power $\mathrm{P}_{\mathrm{k}}$ and the analysis in this case reduces to that of a conventional MIMO system. Therefore in this paper, similar to [3],we assume that there is no additional transmit power constraint at the secondary transmitters and simply assume $P_{T}^{K}=\frac{Q}{\left\|G_{K}\right\|^{2}}$ as the total transmit power at the secondary transmitters. Thus, the proposed results can be interpreted as performance benchmarks for other more realistic signalling schemes.

\section{Performance Analysis}

In this section, we analyze the average capacity and error rate performance of an uplink multiuser SS OSTBC network in closed-form. We also present an analysis of the TAS scheme. To explicitly show the diversity order, we derive some asymptotic results.

\section{A. Multiuser SS OSTBC Network}

For notational simplicity, let $Y_{K}=\left\|G_{K}\right\|_{F}^{2} \quad$ and $U_{K}=\left\|H_{K}\right\|_{F}^{2}$.Then, the received SNR at the $k$ th secondary receiver is expressed as

$$
\gamma_{K}=\frac{Q}{R N Y_{K}}\left\|H_{K}\right\|^{2}{ }_{F}=\frac{Q}{R N Y_{K}} U_{K}
$$

Where $R$ denotes the code rate of STBC. In flat Rayleigh fading channels, $\mathrm{Y}_{\mathrm{k}}$ and $\mathrm{U}_{\mathrm{k}}$ are sums of $\mathrm{N}^{2}$ squared complex Gaussian random variables and both follow the Chi-squared distribution with $2 N^{2}$ degree of freedom. Thus, the probability density function (PDF) of $Y_{k}$ is readily given by

$$
f_{Y_{k}}(\mathrm{y})=\frac{1}{\Gamma\left(\mathrm{N}^{2}\right)} y^{\mathrm{N}^{2}-1} e^{-y}
$$

After collecting $\gamma_{\mathrm{k}}$, the secondary receiver can select the target user according to the rule $k^{*}=\arg \max _{k} \gamma_{\mathrm{k}}$. Based on (3), the PDF and cumulative density function (CDF) of $\gamma_{k}$ are readily given by

And

$$
f_{\gamma_{k}}(\gamma)=\int_{0}^{\infty} \frac{R N_{Y}}{Q} f_{U_{k}}\left(\frac{\gamma R N_{y}}{Q}\right) f_{Y_{k}}(\mathrm{y}) d y
$$

$$
F_{\gamma_{k}}(\gamma)=\frac{\Gamma\left(2 \mathrm{~N}^{2}\right)}{\Gamma^{2}\left(\mathrm{~N}^{2}\right)}\left(\frac{R N}{Q}\right)^{N^{2}} \frac{\gamma^{N^{2}}}{N^{2}}
$$

$$
\times_{2} F_{1}\left(2 N^{2}, N^{2} ; N^{2}+1 ;-\frac{R N}{Q} \gamma\right)
$$

respectively. Eq.(5) is expressed in terms of a Gauss hypergeometric function which makes the analysis difficult. we can now derive the expressions for average capacity and error rate.

\section{1) Average Capacity Analysis}

diversity

The achievable average capacity subject to the OSTBC transmission in a CR network with multiuser is given by 


$$
\begin{aligned}
& C_{K^{*}}^{\text {OSTBC }}=K \log _{2}(e)\left[\frac{\Gamma\left(2 \mathrm{~N}^{2}\right)}{\Gamma^{2}\left(\mathrm{~N}^{2}\right)}\right]^{K} \frac{Q^{N^{2}}}{N^{2(K-1)}} \\
& \sum_{r=0}^{(K-1)\left(N^{2}-1\right)} \cdot \chi_{r}(R N)^{K N^{2}+r-M} \int_{0}^{\infty} \frac{\gamma^{S}}{(\gamma+a)^{M}} \\
& \ln (1+\gamma) d \gamma
\end{aligned}
$$

Where $\mathrm{S}=\mathrm{KN}^{2}+\mathrm{r}-1, \mathrm{M}=(\mathrm{K}+1) \mathrm{N}^{2}+\mathrm{r}$, and $\mathrm{a}=\mathrm{Q} /(\mathrm{RN})$. Define the integral $\mathrm{I}(\mathrm{S}, \mathrm{M}, \mathrm{a})$ as follows:

$$
\mathrm{I}(S, M, a)=\int_{0}^{\infty} x^{S}(x+a)^{-M} \ln (1+x) d x
$$

This integral can be evaluated in closed-form. Now we consider the special case $K=N=1$ which corresponds to the system. Consider in reference [3].Then, $S=0, M=2$ and $a=Q$. Using [13, Eq.(7)], we can easily verify that the capacity expression (6) reduces to [3, Eq.(24)]. Thus, as a double check, the correctness of our analysis is further verified.

\section{2) Bit-Error Rate Analysis}

We can now analyze the BER performance based on the CDF-based method. The CDF can be expressed with the help of [13,Eq.(7)] and [14] as follows:

$$
\begin{gathered}
f_{\gamma k^{*}}(\gamma)=K\left[\frac{\Gamma\left(2 \mathrm{~N}^{2}\right)}{\Gamma^{2}\left(\mathrm{~N}^{2}\right)}\right]^{K} \frac{(R N)^{K N^{2}}}{N^{2(K-1)}} \sum_{r=0}^{(k-1)\left(N^{2}-1\right)} \sum_{s=0}^{N^{2}-1} \chi_{r} \\
\frac{\left(-\left(N^{2}-1\right)\right)_{S}(\boldsymbol{S}+1)_{s}(\boldsymbol{R} N)^{r+s}}{(\boldsymbol{S}+2)_{s} s !(\boldsymbol{S}+\mathbf{1})}\left(\frac{\gamma}{R N_{\gamma+Q}}\right)^{S+1+s}
\end{gathered}
$$

where $S$ was introduced in (6). The BER

performance can be computed in terms of the CDF by introducing a standard normal distribution RV $V$. Namely,

$$
P_{e}=E_{V}\left[F_{\gamma K^{*}}\left(\frac{V^{2}}{2}\right)\right]
$$

By substituting (8) into (9) and taking advantage of [13,Eq.(3)], we obtain

$$
\begin{aligned}
& P_{e}^{O S T B C}=\frac{K}{2 N^{2(K-1)}} \sqrt{\frac{1}{\pi}}\left[\frac{\Gamma\left(2 \mathrm{~N}^{2}\right)}{\Gamma^{2}\left(\mathrm{~N}^{2}\right)}\right]^{K} \sum_{r=0}^{(k-1)\left(N^{2}-1\right)} \sum_{s=0}^{N^{2}-1} \chi_{r} \\
& \frac{\left(-\left(N^{2}-1\right)\right)_{S}(S+1)_{s}}{(S+2)_{s} \mathrm{~s}(\mathrm{~S}+\mathbf{1})} \Gamma(\mathrm{S}+\mathrm{s} 1.5)\left(\frac{\mathrm{Q}}{\mathrm{RN}}\right)^{-(\mathrm{S}+1+\mathrm{s})} \\
& .2^{\mathrm{F}_{0}}\left(\mathrm{~S}+\mathrm{s}+1.5, \mathrm{~S}+1+\mathrm{s} ;-\frac{R N}{Q}\right) \quad(10)
\end{aligned}
$$

where $\mathrm{pFq}(\mathrm{a} 1, \ldots, \mathrm{ap} ; \mathrm{b} 1, \ldots, \mathrm{bq} ; \mathrm{z})$ denotes the generalized hypergeometric function. Notice that the property $2 \mathrm{~F} 0(\mathrm{p}, \mathrm{q} ; ;-1 / \mathrm{x})=\mathrm{x}^{\mathrm{p}} \psi(\mathrm{p}, \mathrm{p}-\mathrm{q}+1, \mathrm{x})$ eq 9 was used to drive eq 10 . While being exact, the above closed-form expression does not provide much information about the diversity order. Therefore, we need to resort to an approximate analysis. Using Taylor series to expand (4) with respect to $\gamma$ around zero, the CDF (5) can be approximated as

$F_{\gamma_{k}}(\gamma) \approx \frac{\Gamma\left(2 \mathrm{~N}^{2}\right)}{\Gamma^{2}\left(\mathrm{~N}^{2}\right) \mathrm{N}^{2}}\left(\frac{R N}{Q}\right)^{N^{2}} \gamma^{N^{2}}(11)$

Therefore, the approximate PDF for $\gamma k^{*}$ is given by

$$
f_{\gamma k^{*}}(\gamma) \approx K\left[\frac{\Gamma\left(2 \mathrm{~N}^{2}\right)}{\Gamma^{2}\left(\mathrm{~N}^{2}\right)}\right]^{K}\left(\frac{R N}{Q}\right)^{K N^{2}} \frac{\gamma^{K N^{2}-1}}{N^{2(k-1)}}
$$


and the resulting approximate $\mathrm{CDF}$ is given by,

$$
F_{\gamma k^{*}}(\gamma) \approx\left[\frac{\Gamma\left(2 \mathrm{~N}^{2}\right)}{\Gamma^{2}\left(\mathrm{~N}^{2}\right)}\right]^{K}\left(\frac{R N}{Q}\right)^{K N^{2}} \frac{\gamma^{K N^{2}}}{N^{2 k}}
$$

Similar to the analysis for (12), we can obtain the approximate BER expression as

$$
P_{e}^{O S T B C} \approx\left[\frac{\Gamma\left(2 \mathrm{~N}^{2}\right)}{\Gamma^{2}\left(\mathrm{~N}^{2}\right)}\right]^{K}\left(\frac{R N}{Q}\right)^{K N^{2}} \frac{\Gamma\left(K N^{2}+0.5\right)}{2 N^{2 k} \sqrt{\pi}}
$$

Expression (14) indicates that a multiuser OSTBC CR network can achieve the diversity order $\mathrm{KN}^{2}$. In traditional down-link multiuser diversity MIMO systems, the performance of applying transmit antenna selection is better than that of the transmit diversity system. Thus, a legitimate question is whether a similar result holds for the considered SS system. Next, we will derive the performance expressions for the transmit antenna selection scheme.

\section{B. TAS Scheme}

For a TAS scheme, we assume that only one transmit antenna is selected for transmit signals at the secondary transmitters and the secondary receiver applies the maximum ratio combining (MRC). As a result, the effective SNR $\gamma_{k}$ of the secondary transmitter $k$ with respect to its transmit antenna $\mathrm{j}$ at the combiner output of the secondary receiver. Therefore, the decision rule at the secondary receiver becomes

$$
k^{*}=\arg \max _{k, j} \frac{Q}{Y_{j}^{k}} \sum_{i=1}^{N}\left|h_{i, j}^{k}\right|^{2}
$$

For Rayleigh fading channels, both $\sum_{i=1}^{N}\left|h_{i, j}^{k}\right|^{2}$ and $Y_{j}^{k}$ admit a Chi-squared distribution with $2 N$-degrees of freedom. Similar to the analysis in the Subsection A, we express the PDF and CDF of $\gamma_{k}$ as follows:

And

$$
f_{\gamma_{k}}(\gamma)=\frac{\Gamma(2 \mathrm{~N})}{\Gamma^{2}(\mathrm{~N}) \mathrm{Q}^{\mathrm{N}}} \gamma^{N-1}\left(\frac{\gamma}{Q}+1\right)^{-2 N}
$$

$f_{\gamma_{k}}(\gamma)=\frac{\Gamma(2 \mathrm{~N})}{\Gamma(\mathrm{N}+1)} \sum_{t=0}^{N-1} \frac{(-(N-1))_{t}(N)_{t}}{\Gamma(\mathrm{N})(\mathrm{N}+1)_{\mathrm{t}} \mathrm{t} !}\left(\frac{\gamma}{\gamma+Q}\right)^{N+t}$

respectively. Using (16) and (17), the PDF of $\gamma k^{*}$ is further expressed as:

$f_{\gamma k^{*}}(\gamma)=\frac{K N Q^{N}}{N^{K N-1}}\left[\frac{\Gamma(2 \mathrm{~N})}{\Gamma(\mathrm{N}+1)}\right]^{K N} \frac{\gamma^{K N^{2}-1}}{(\gamma+Q)^{K N^{2}+N}} \times$

$\sum_{r=0}^{(K N-1)(N-1)} \bar{\omega}_{r}\left(\frac{\gamma}{\gamma+Q}\right)^{r}$

Where $\bar{\omega}_{r}$ is the coefficient of $\left(\frac{\gamma}{\gamma+Q}\right)^{r}$

in the expansion of

Using (7) and (18), we obtain the system throughput of the TAS scheme in a SS network as

$C_{K^{*}}^{T A S}=\log _{2}(e) \frac{K N Q^{N}}{N^{K N-1}}\left[\frac{\Gamma(2 \mathrm{~N})}{\Gamma(\mathrm{N}+1)}\right]^{K N} \sum_{r=0}^{(K N-1)(N-1)} \bar{\omega}_{r} I\left(K N^{2}+r-1, K N^{2}+N+r, Q\right)$

For comparison purposes, we also derive the approximate BER expression

$P_{e}^{T A S} \approx\left[\frac{\Gamma(2 \mathrm{~N})}{\Gamma^{2}(\mathrm{~N}) \mathrm{Q}^{\mathrm{N}}}\right]^{K N} \frac{\Gamma\left(\mathrm{KN}^{2}+0.5\right)}{2 N^{K N} \sqrt{\pi}}$

turns out that the TAS scheme achieves also the diversity order $K N^{2}$. Based on (14) and (20), we observe that the ratio $P_{e}^{T A S} / P_{e}^{O S T B C}$ is smaller than 1 when $N \geq 2$. For example, $P_{e}^{T A S} \approx 0.0161^{K} P_{e}^{O S T B C}$ when $N=2$. 


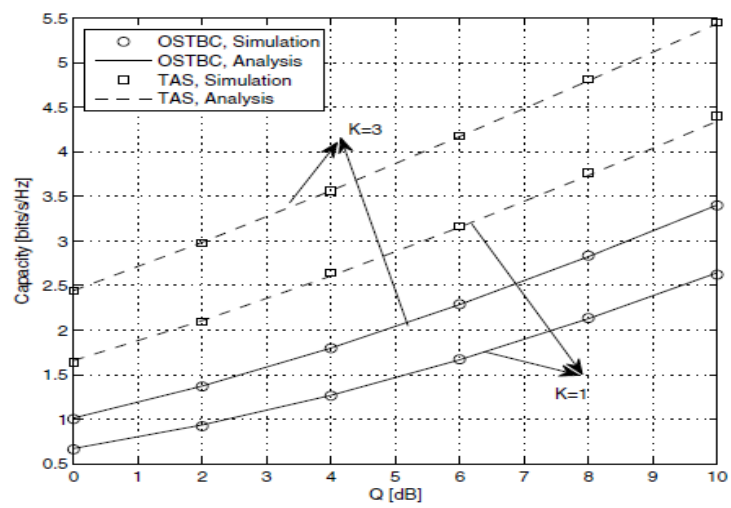

Fig. 2. Capacity comparison between OSTBC and TAS SS network with multiuser diversity.

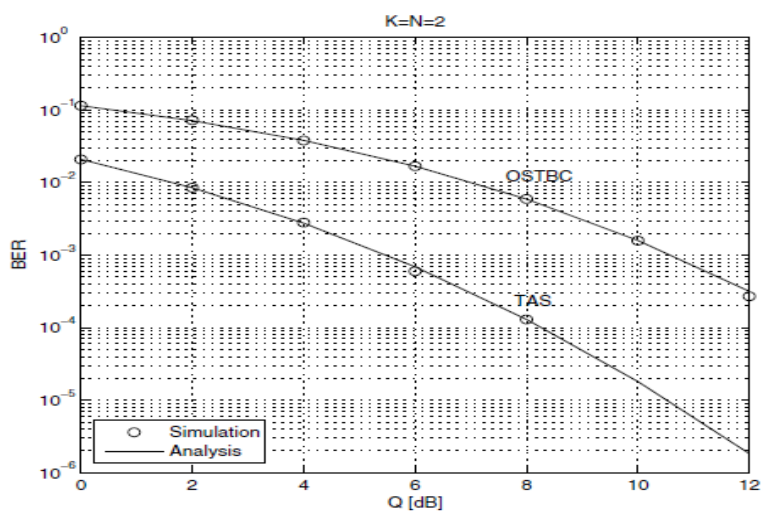

Fig. 3. BER performance comparison between OSTBC and TAS

\section{Numerical Results And Conclusions}

This section describes several numerical simulation results to verify the proposed analysis. The number of channelMonte- Carlo realizations is $10^{5}$. We consider the uncoded BPSK modulation and the Alamouti signaling scheme, e.g., $N=2$.

Fig. 2 shows the average throughput comparison between OSTBC and TAS in a multiuser diversity SS system with $K=1$ and 3. As expected, the simulation results coincide with the analytical ones, and the multiuser diversity gain can further improve the system performance. Also, we found that the TAS scheme can achieve higher capacity. Fig. 3 shows the BER comparison between the TAS and OSTBC schemes for $K=2$. It clearly shows that the TAS scheme can achieve better performance. This fact is in agreement with the similar result observed in traditional multiuser diversity MIMO systems. However, it should be noted that in such a system the TAS scheme requires the secondary receiver to indicate the transmitter which antenna is selected, which increases the system complexity. Therefore, there is a tradeoff between the system complexity and performance.

\section{References}

[1] A.Ghasemi and E.S.Sousa, "Fundamental limits of spectrum-sharing in fading environments," IEEE Trans. Wireless Commun., vol. 6, no. 2, pp. 649-658, Feb. 2007.

[2] T. W. Ban, W. Choi, B. C. Jung, and D. K. Sung, "Multiuser diversity in a spectrum sharing system," IEEE Trans. Wireless Commun., vol. 8, no. 1, pp. 102-106, Jan. 2009

[3] H. A. Suraweera, P. J. Smith, and M. Shafi, "Capacity limits and performance analysis of cognitive radio with imperfect channel knowledge," IEEE Trans. Veh. Technol., vol. 59, no. 4, pp. 1811-1822, May 2010.

[4] Z. Rezki and M.-S. Alouini, "Ergodic capacity of cognitive radio under imperfect channel state information," IEEE Trans. Veh. Technol., vol. 61, no. 5, pp. 2108-2119, June 2012.

[5] R. Zhang and Y.-C. Liang, "Exploiting multi-antennas for opportunistic spectrum sharing in cognitive radio networks," IEEE J. Sel. Areas Signal Process., vol. 2, no. 1, pp. 88-102, Feb. 2008.

[6] Y. Li and A. Nosratinia,"Capacity limits of multiuser multiantenna cognitive networks," IEEE Trans. Inf. Theory, vol. 58, no. 7, pp. 4493- 4508, July 2012.

[7] C. J. Chen L. C. Wang, "A unified capacity analysis for wireless systems with joint multiuser scheduling and antenna diversity in Nakagami fading channels," IEEE Trans. Commun., vol. 54, pp. 469-478, Mar. 2006. 